07;09

\title{
Экспериментальные исследования динамики распространения включенного состояния низковольтных лазеров-тиристоров на основе гетероструктур AIGaAs/InGaAs/GaAs
}

\author{
(C) П.С. Гаврина ${ }^{1}$, О.С. Соболева ${ }^{1}$, А.А. Подоскин ${ }^{1}$, Д.Н. Романович ${ }^{1}$, В.С. Головин ${ }^{1}$, С.О. Слипченко ${ }^{1}$, \\ Н.А. Пихтин ${ }^{1}$, Т.А. Багаев ${ }^{2}$, М.А. Ладугин ${ }^{2}$, А.А. Мармалюк ${ }^{2}$, В.А. Симаков ${ }^{2}$ \\ ${ }^{1}$ Физико-технический институт им. А.Ф. Иофффе РАН, Санкт-Петербург, Россия \\ ${ }^{2}$ АО „Научно-исследовательский институт „Полюс“ им. М.Ф. Стельмаха“, Москва, Россия \\ E-mail: gavrina@mail.ioffe.ru
}

Поступило в Редакцию 28 декабря 2018г.

В окончательной редакции 23 января 2019г.

Принято к публикации 25 января 2019г.

\begin{abstract}
Предложена методика определения пространственно-временно́й динамики тока в полупроводниковых гетероструктурах, основанная на модуляции внешнего излучения при прохождении через исследуемый кристалл. Апробация методики проведена на полупроводниковых лазерах-тиристорах на основе гетероструктур $\mathrm{AlGaAs} / \mathrm{InGaAs} / \mathrm{GaAs}$. Продемонстрировано качественное совпадение результатов эксперимента с результатами предыдущих измерений пространственно-временно́й динамики в приборе.
\end{abstract}

DOI: 10.21883/PJTF.2019.08.47612.17662

Работа сильноточных приборов зачастую сопровождается локализацией тока с формированием токовых шнуров, что приводит к перегреву структуры и понижению надежности прибора. Экспериментальные методы исследования шнурования тока в структурах базируются на регистрации изменения термических, электрических или оптических характеристик прибора. Методика обнаружения локального повышения температуры в приборе с помощью тепловизионных камер (см., например, [1]) имеет пространственное разрешение на уровне десятков микрометров, временно́е разрешение обычно отсутствует. Метод 2D TIM (transient interference mapping) [2-4] использует эффект изменения фазы оптического луча при прохождении через полупроводник и отражении от внешней поверхности прибора. Временно́е разрешение методики определяется шириной оптического импульса. Возникновение и исчезновение доменов сильного поля можно отследить с помощью анализа токовой динамики (см., например, [5]) и вольт-амперных характеристик приборов, что было показано в работах [6-9]. Однако возможности электрических методов исследования структур с распространением включенного состояния ограничены. В $[6,8,10]$ положение токовых шнуров определяется по спонтанному излучению в области с повышенной плотностью тока, однако такой способ может иметь меньшую эффективность при работе с приборами на основе непрямозонных полупроводниковых материалов.

В настоящей работе предлагается методика измерения пространственно-временно́й токовой динамики в полупроводниковых гетероструктурах, основанная на эффектах модуляции диагностического оптического излучения при прохождении через область с высокой плотностью тока. За основу взят подход из работы [11], где оце- ниваются оптические потери в волноводе мощного полупроводникового лазера с шириной полоска $100 \mu \mathrm{m}$ по модуляции проходящего через волновод внешнего излучения от полупроводникового лазера с такой же шириной полоска поглощением на свободных носителях заряда. Нашим объектом исследования был выбран лазертиристор (ЛТ) полосковой конструкции (ширина полоска $200 \mu \mathrm{m})$, содержащий в одном кристалле оптопару лазерный диод-фототранзистор (см. [12]). Схематически конструкция, составы слоев и электрическая схема ЛТ представлены на рис. 1. На анод и катод подается постоянное напряжение, импульс управления амплитудой десятки-сотни миллиампер запускает инжекцию носителей заряда в лазерную часть. Собственное спонтанное излучение ЛТ частично поглощается в слаболегированной базе $p$-GaAs, создавая начальные носители заряда для процесса ударной ионизации. В [13] показано, что комбинация эффектов фотогенерации и ударной ионизации в узкой области сильного поля вблизи коллекторного $p-n$-перехода приводит к быстрому переводу ЛТ в состояние с низким дифференциальным сопротивлением, т.е. во включенное состояние. Однако флуктуации плотности тока в параллельной эпитаксиальным слоям плоскости (в том числе возникающие из-за неоднородной фотогенерации носителей заряда в базе $p$-GaAs) в условиях наличия ударной ионизации способствуют формированию токовых шнуров, проходящих перпендикулярно слоям гетероструктуры. В работе [10] по динамике собственного излучения из лазерной части были обнаружены области начального включения ЛТ, располагающиеся вблизи контактов управления. В связи с этим целесообразно провести сравнение результатов настоящей работы с результатами [10]. 

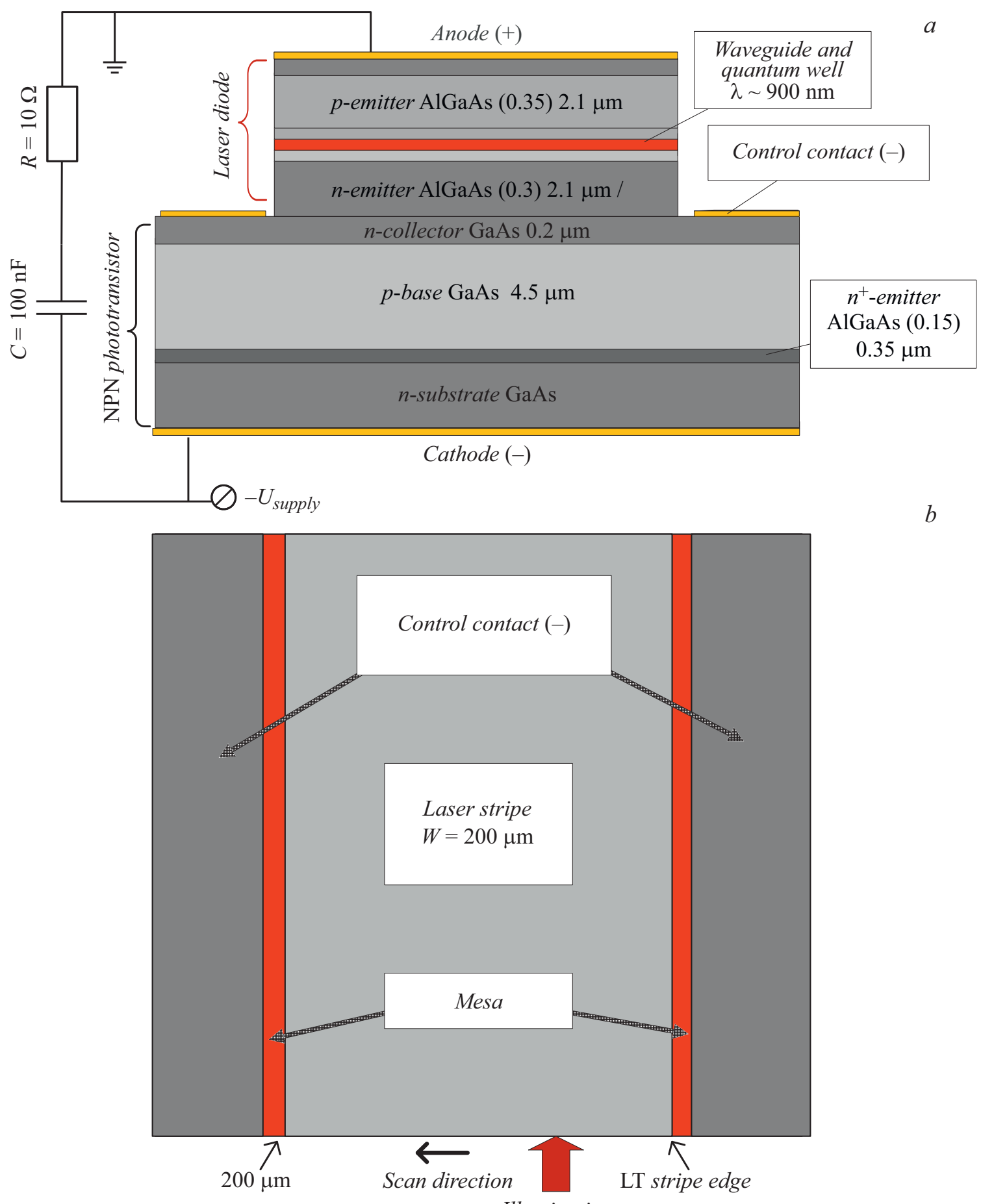

$b$

Illumination

Рис. 1. Схематическое изображение лазера-тиристора. $a-$ вид со стороны торца кристалла ЛТ, $b-$ вид сверху.

В эксперименте ввод излучения осуществлялся в базовую область $p$-GaAs в направлении оси резонатора (рис. $1, b$ ) с помощью оптической схемы, представленной на рис. 2,a. В качестве источников диагностического излучения использовались два типа полупроводниковых лазеров полосковой конструкции, излучающих в спектральном диапазоне, для которого слой базы $p$-GaAs оптически прозрачен. Для первой демонстрации на- личия модуляции (по аналогии с упомянутой выше работой [11]) проходящего через кристалл излучения был выбран лазер-источник с шириной полоска $100 \mu \mathrm{m}$ $(\lambda \sim 1060 \mathrm{~nm})$ при ширине полоска ЛТ $200 \mu \mathrm{m}$. Однако для получения пространственного разрешения методики было необходимо принимать меры по уменьшению апертуры лазера-источника. Для проведения пространственного сканирования вдоль торца кристалла ЛТ 


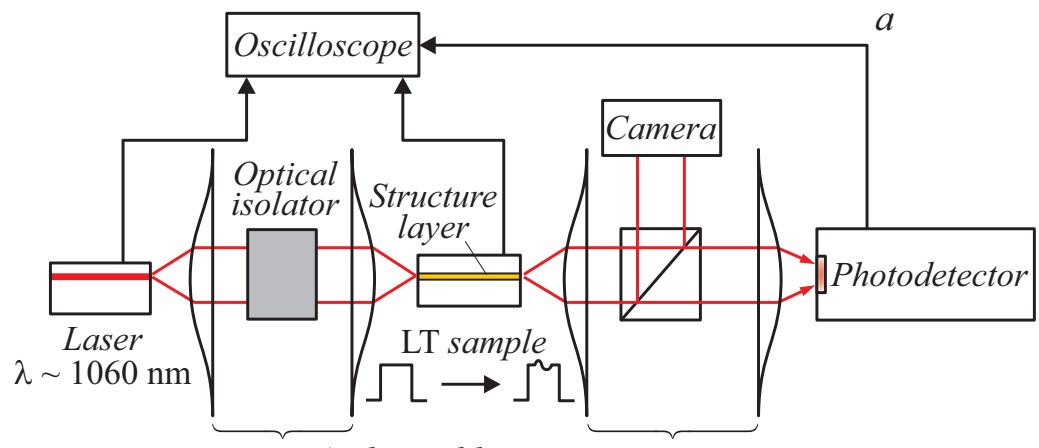

Aspherical lens system
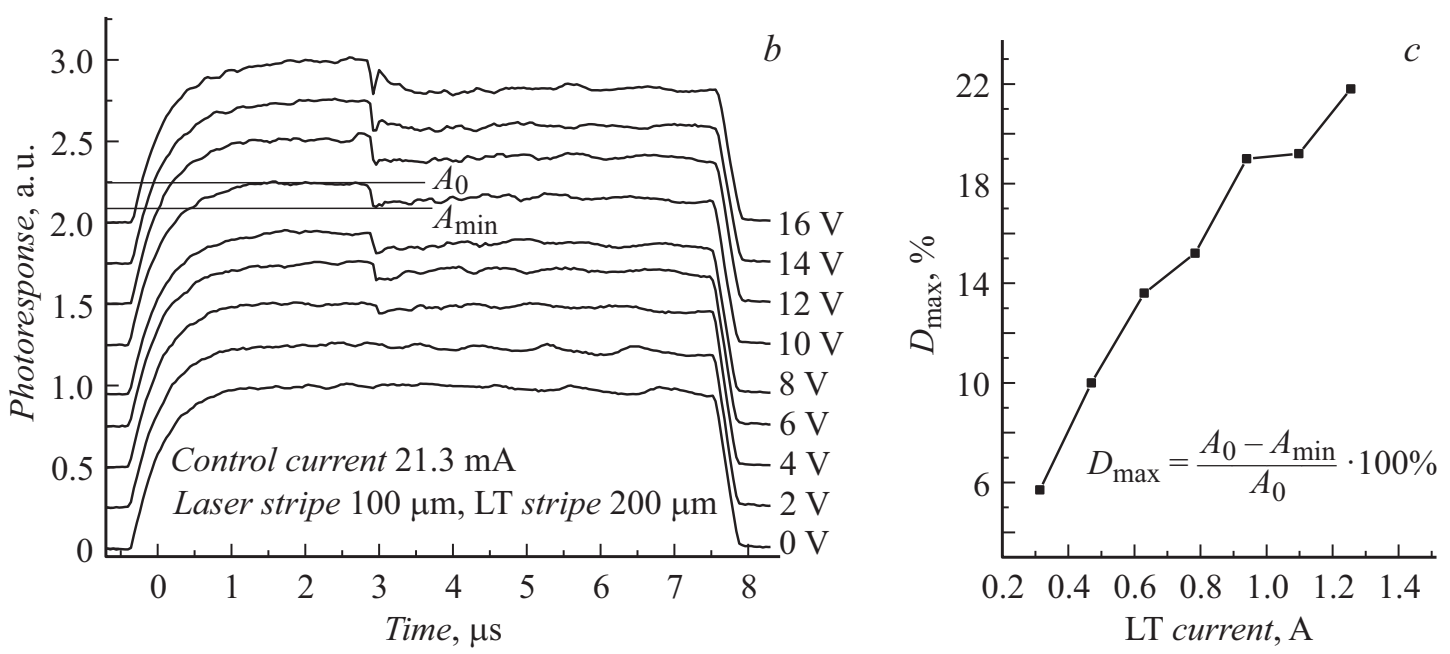

Рис. 2. $a-$ оптическая схема эксперимента; $b-$ фотоотклик от излучения многомодового лазера с шириной полоска $100 \mu$ m, прошедшего через базовую область ЛТ, при напряжениях питания от 0 до $16 \mathrm{~V} ; c$ - зависимость максимальной глубины модуляции $D_{\max }$ прошедшего через ЛТ излучения от амплитуды протекающего через ЛТ тока.

применялся лазер-источник с шириной полоска $5 \mu \mathrm{m}$ $(\lambda \sim 1128 \mathrm{~nm})$, что позволило получить на выходе из кристалла ЛТ длиной $1 \mathrm{~mm}$ апертуру пучка менее $20 \mu \mathrm{m}$. При этом сканирование осуществлялось посредством перемещения кристалла ЛТ, размещенного на подвижном столике, относительно оптической оси лазераисточника.

На рис. 2,b представлены временны́е зависимости фотооткликов от прошедшего через ЛТ излучения от лазера-источника с шириной полоска $100 \mu \mathrm{m}$ при различных амплитудах протекающего через ЛТ тока. Отчетливо видна отрицательная модуляция амплитуды излучения на фоне прямоугольного импульса при приложенных напряжениях, превышающих 4 V. Как и в работе [11], модуляция может быть связана с поглощением диагностического излучения на свободных носителях заряда, при этом амплитуда модуляции пропорциональна плотности протекающего тока. За максимальную глубину модуляции $D_{\max }$ примем разность между амплитудой первоначального уровня оптического сигнала $A_{0}$ (в ходе эксперимента оставалась постоянной) и минимальной амплитудой оптического сигнала $A_{\min }$ (для наглядности уровни $A_{0}$ и $A_{\min }$ на рис. $2, b$ показаны горизонтальными линиями для напряжения $10 \mathrm{~V})$. Измеряя протекающий через ЛТ импульсный ток, можно построить зависимость максимальной глубины отрицательной модуляции $D_{\max }$ от амплитуды тока через ЛТ (рис. 2, c). Из рис. 2, $c$ видно, что глубина модуляции может превышать 20\% от первоначального уровня сигнала.

Рис. 3 показывает набор фотооткликов от прошедшего через кристалл ЛТ излучения лазера-источника с шириной полоска $5 \mu \mathrm{m}$ при пространственном сканировании в направлении от края полоска (вблизи контакта управления, нижний сигнал на рис. 3) к центру (верхний сигнал на рис. 3). Первоначальный уровень оптического сигнала показан горизонтальными штриховыми линиями для каждой пространственной координаты. Видно, что форма и глубина отрицательной модуляции различаются от одной координаты засветки к другой, что свидетельствует о существенно неоднородном протекании тока в ЛТ. Максимальные глубина отрицательной модуляции и скорость ее нарастания наблюдаются со стороны контакта управления, в то время как модуляция при засветке центральных участков торца ЛТ имеет меньшие фронт нарастания и амплитуду относительно первоначального уровня оптического сигнала. Можно 


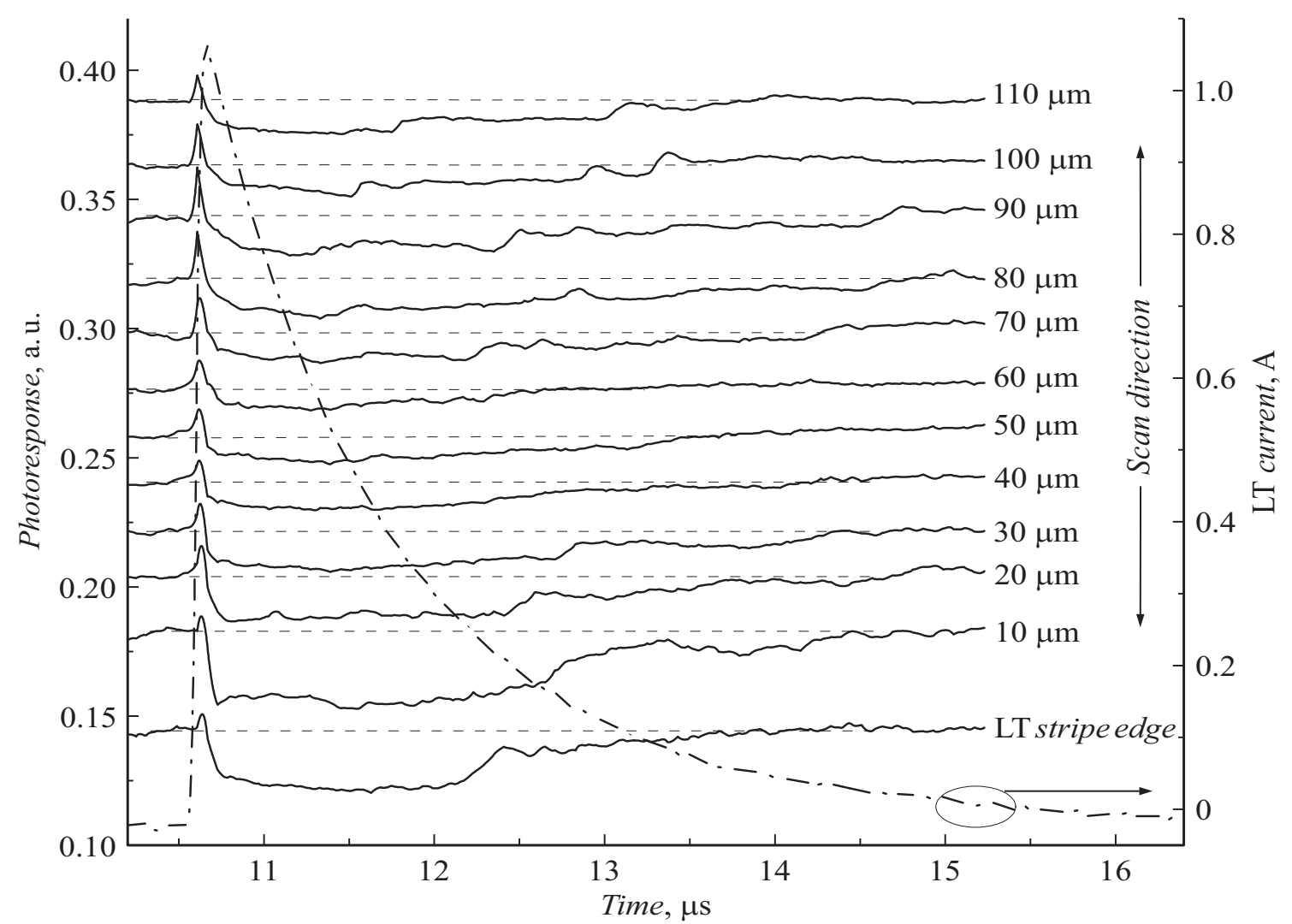

Рис. 3. Набор фотооткликов (сплошные линии) от излучения лазера с шириной полоска $5 \mu$ m после прохождения базовой области ЛТ при пространственном сканировании образца в направлении от края кристалла (LT stripe edge) к центру до точки $110 \mu \mathrm{m}$ (штриховые линии - начальный уровень оптического сигнала) и импульс протекающего через ЛТ тока (штрихпунктирная линия, правая шкала).

сделать вывод о наличии шнурования тока на начальных этапах включения ЛТ вблизи контакта управления, что качественно повторяет результаты работы [10]. При этом можно отметить, что диаметр токового шнура в момент времени с максимальной плотностью тока не превышает $20 \mu \mathrm{m}$.

Итак, в работе предложена методика исследования пространственно-временно́й токовой динамики в полупроводниковых приборах на основе эпитаксиальных гетероструктур, основанная на модуляции излучения при прохождении через кристалл. Испытания методики на низковольтных ЛТ показали качественное совпадение результатов с предыдущими оценками пространственной динамики собственного излучения ЛТ из работы [10]. Однако по сравнению с подходом [10] предлагаемая методика является более универсальной, поскольку не требует наличия собственного излучателя в гетероструктуре. Ее пространственное разрешение определяется точностью позиционирования кристалла относительно максимума оптического пучка и расходимостью пучка внутри кристалла и составляет на данный момент $10 \mu \mathrm{m}$. Временно́е разрешение, задаваемое характеристиками измерительного оборудования, составляет 1 ns. Кроме того, по общему снижению амплитуды проходящего излучения можно судить о степени поврежденности прибора вследствие образования дефектов в местах повышенной плотности тока. Дальнейшая работа будет направлена на совершенствование предлагаемой методики с целью повышения ее информативности.

П.С. Гаврина и О.С. Соболева выражают благодарность Российскому фонду фундаментальных исследований (договор № 18-38-00906 „Закономерности формирования и развития пространственных неоднородностей тока в низковольтных полупроводниковых гетероструктурах с нелинейной обратной связью“).

\section{Список литературы}

[1] Hempel M., Tomm J.W. // Novel in-plane semiconductor lasers XIII. International Society for Optics and Photonics, 2014. V. 9002. P. 90021 H. DOI: $10.1117 / 12.2035488$

[2] Pogány D., Dubec V., Bychikhin S., Furbock C., Litzenberger A., Groos G., Stecher M., Gornik E. // IEEE Electron Dev. Lett. 2002. V. 23. N 10. P. 606-608. DOI: $10.1109 /$ LED.2002.803752

[3] Pogany D., Bychikhin S., Furbock C., Litzenberger M., Gornik E., Groos G., Esmark K., Stecher M. // IEEE Trans. Electron Dev. 2002. V. 49. N 11. P. 2070-2079. DOI: 10.1109/TED.2002.804724 
[4] Haberfehlner G., Bychikhin S., Dubec V., Heer M., Podgaynaya A., Pfost M., Stecher M., Gornik E., Pogany D. // Microelectron. Reliab. 2009. V. 49. N 9-11. P. 1346-1351.

DOI: $10.1016 /$ j.microrel.2009.07.032

[5] Левинштейн М.Е., Пожела Ю.К., Шур М.С. Эффект Ганна. М.: Сов. радио, 1975. $288 \mathrm{c.}$

[6] Кернер Б.С., Синкевич В.Ф. // Письма в ЖЭТФ. 1982. T. 36. B. 10. C. 359-362.

[7] Сергеев В.А., Куликов А.А. // Радиоэлектронная техника. 2012. № 1. C. 66-72.

[8] Вайнштейн С.Н., Жиляев Ю.В., Левинштейн М.Е. // Письма в ЖТФ. 1988. Т. 14. В. 16. С. 1526-1530.

[9] Vainshtein S., Yuferev V., Palankovski V., Ong D.S., Kostamovaara J. // Appl. Phys. Lett. 2008. V. 92. N 6. P. 062114. DOI: $10.1063 / 1.2870096$

[10] Slipchenko S.O., Podoskin A.A., Soboleva O.S., Pikhtin N.A., Bagaev T.A., Ladugin M.A., Marmalyuk A.A., Simakov V.A., Tarasov I.S. // J. Appl. Phys. 2017. V. 121. N 5. P. 054502. DOI: $10.1063 / 1.4975411$

[11] Веселов Д.А., Пихтин Н.А., Лютеикий А.В., Николаев Д.Н., Слипченко С.О., Соколова З.Н., Шамахов В.В., Шашкин И.С., Воронкова Н.В., Тарасов И.С. // Квантовая электроника. 2015. Т. 45. № 7. С. 604-606.

[12] Slipchenko S.O., Podoskin A.A., Pikhtin N.A., Tarasov I.S., Gorbatyuk A.V. // IEEE Trans. Electron Dev. 2015. V. 62. N 1. P. 149-154. DOI: 10.1109/TED.2014.2372317

[13] Yuferev V.S., Podoskin A.A., Soboleva O.S., Pikhtin N.A., Tarasov I.S., Slipchenko S.O. // IEEE Trans. Electron Dev. 2015. V. 62. N 12. P. 4091-4096.

DOI: $10.1109 /$ TED.2015.2483371 